\title{
Increasing the Accuracy of Digital Force Control Process Using the Act-and-Wait Concept
}

\author{
Tamás Insperger, László L. Kovács, Péter Galambos, and Gábor Stépán
}

\begin{abstract}
Proportional gains are to be increased in force control processes in order to reduce the force error. However, the control process may become unstable for large gains due to the digital and delay effects. In this paper, the act-and-wait control concept is compared with the traditional, continuous control concept for a digital force control model with proportional feedback subject to a short, one sample unit feedback delay. Both concepts are implemented in an experimental setup. It is shown that the proportional gain can be increased significantly without losing stability when the actand-wait controller is used; thus, the force error can effectively be decreased this way. The results are confirmed by experiments.
\end{abstract}

Index Terms-Discrete-time systems, feedback systems, force control, stability.

\section{INTRODUCTION}

$\mathbf{F}$ ORCE control is a frequent mechanical controlling problem in robotics since most robotic applications involve interactions with other objects. The first papers on the basics of force control approaches appeared in the early 1980s starting with the pioneering work of Whitney [1], Mason [2], and Raibert and Craig [3]. Since then, several comprehensive textbooks have been published summarizing different methods of force control processes in the field of robotics [4]-[6]. The aim of force control is to provide a desired force between the actuator and the environment (or workpiece). In order to achieve high accuracy in maintaining the prescribed contact force against the Coulomb friction, high proportional control gains are to be used [4], [5]. However, in practical realizations of force control processes with high proportional gains, the robot often loses stability, and starts to oscillate at a relatively low frequency. These oscillations are mainly caused by the digital effects [1] and by the time delays in the feedback loop [7], [8]. Such delays arise due to the time required for the computer to compute the control

Manuscript received November 18, 2008; revised April 11, 2009. First published July 6, 2009; current version published March 31, 2010. Recommended by Technical Editor W.-J. Kim. The work of T. Insperger was supported in part by the Hungarian Academy of Sciences under the János Bolyai Research Scholarship and in part by the Hungarian National Science Foundation under Grant K72911. The work of G. Stépán was supported in part by the Hungarian National Science Foundation under Grant OTKA T068910.

T. Insperger and G. Stépán are with the Department of Applied Mechanics, Budapest University of Technology and Economics, H-1521 Budapest, Hungary (e-mail: inspi@mm.bme.hu; stepan@mm.bme.hu).

L. L. Kovács is with the Hungarian Academy of Sciences (HAS)-Budapest University of Technology and Economics (BUTE) Research Group on Dynamics of Machines and Vehicles, H-1521 Budapest, Hungary (e-mail: kovacs@mm.bme.hu).

P. Galambos is with the Department of Manufacturing Science and Technology, Budapest University of Technology and Economics, H-1521 Budapest, Hungary (e-mail: galambos@manuf.bme.hu).

Color versions of one or more of the figures in this paper are available online at http://ieeexplore.ieee.org.

Digital Object Identifier 10.1109/TMECH.2009.2024683 force. In addition, the control signal produced by the computer is piecewise constant (zero-order hold) function of time.

Time delays are inherent attributes of feedback systems that usually have unfavorable effects on the performance of the control process. Teleoperation is a typical example, where communication delay plays a crucial role [9], [10], but similar delays may arise in haptic interfaces as well [11]. The problem with time-delayed systems is that the dimension of their phase space is usually larger than the dimension of the state variables; therefore, the number of poles to be controlled is usually larger than the number of control parameters. Thus, complete pole placement is not possible for these systems using traditional constant feedback gains. The act-and-wait control concept is an effective tool to deal with pole placing for systems with feedback delay. The act-and-wait technique was introduced in [12] for discretetime systems and in [13] for continuous-time systems. The crux of the technique is that the controller is periodically switched on and off with the switch-off period being larger than the feedback delay. Using this periodic switching, the extra poles due to the time delay are automatically assigned to zero, and the pole placement problem of the remaining poles is possible if certain conditions are fulfilled for the system parameters.

Although theoretical predictions showed that the act-and-wait method can effectively be used for discrete-time control systems with feedback delay [12], it has never been confirmed by experiments until now. In this paper, a digital force control process with a short (one sample unit) feedback delay is implemented in an experimental setup. A proportional feedback is applied in order to decrease the force error. It is shown that the proportional gain can be increased, and consequently, the force error can be decreased, without losing stability if the feedback is periodically switched on and off according to the act-and-wait concept. The structure of the paper is as follows. First, in Section II, the act-and-wait concept is summarized briefly for discrete-time systems based on [12]. Then, in Section III, the experimental setup and the corresponding mechanical model are presented for the continuous and for the act-and-wait control concept. In Section IV, the experimental results are compared to the theoretical predictions. The paper is concluded in Section V.

\section{THE ACT-AND-WAIT CONCEPT}

Consider the discrete-time system

$$
\mathbf{x}(j+1)=\mathbf{A} \mathbf{x}(j)+\mathbf{B u}(j-R)
$$

with the controller

$$
\mathbf{u}(j)=\mathbf{D} \mathbf{x}(j)
$$


where $\mathbf{x} \in \mathbb{R}^{n}$ is the state, $\mathbf{u} \in \mathbb{R}^{m}$ is the input, $\mathbf{A} \in \mathbb{R}^{n \times n}, \mathbf{B} \in$ $\mathbb{R}^{n \times m}, \mathbf{D} \in \mathbb{R}^{m \times n}$ are constants and $j \in \mathbb{Z}$. We assume that the feedback delay $R \in \mathbb{Z}^{+}$is a fixed parameter of the system that cannot be adjusted during the control design. State augmentation of system (1) with controller (2) yields the discrete map

$$
\mathbf{z}(j+1)=\Psi \mathbf{z}(j)
$$

with $\mathbf{z}(j)=\left(\mathbf{x}^{T}(j), \mathbf{u}^{T}(j-1), \ldots, \mathbf{u}^{T}(j-R)\right)^{T} \in \mathbb{R}^{n+m R}$. Here, the coefficient matrix

$$
\Psi=\left(\begin{array}{ccccc}
\text { A } & 0 & \ldots & 0 & \text { B } \\
\text { D } & 0 & \ldots & 0 & 0 \\
0 & \text { I } & \ldots & 0 & 0 \\
\vdots & & \ddots & & \vdots \\
0 & 0 & \ldots & \text { I } & 0
\end{array}\right)
$$

is actually the $(n+m R) \times(n+m R)$ monodromy matrix of the system [14], [15]. The identity submatrices I below the diagonal of $\Psi$ represent the delay effect in the feedback. Stability properties are determined by the eigenvalues of $\boldsymbol{\Psi}$, which are also called characteristic multipliers or poles. The system is asymptotically stable if all the $(n+R m)$ poles lie in the open unit disk of the complex plane. It can easily be seen that in general cases, the poles cannot be controlled completely by the control parameters, i.e., by the elements of matrix $\mathbf{D}$. This also causes problems during the stabilization of the process.

The act-and-wait control concept for discrete-time systems was introduced in [12]. It is a special case of periodic controllers where the control is periodically switched on and off in the form

$$
\mathbf{u}(j)=g(j) \mathbf{D} \mathbf{x}(j)
$$

where $g(j)$ is the $K$-periodic switching function defined as

$$
g(j)= \begin{cases}1, & \text { if } j=h K, h \in \mathbb{Z} \\ 0, & \text { otherwise. }\end{cases}
$$

Here, integer $K$ is called period parameter. While the feedback delay $R$ is a given system parameter, the period parameter $K$ can be chosen during the control design.

If $K=1$, then $g(j) \equiv 1$. In this case, the control is continuously active, which corresponds to controller (2).

If $K \geq 2$, then $g(j)$ alternates between 1 and 0 . In the first discrete step, $g(j)=1$ and the control is active (act), while in the following $(K-1)$ number of steps, $g(j)=0$, and the control term is switched off (wait), then in the $(K+1)$ th step, the control is active again, etc. In [12], it was shown that if the period parameter $K$ is chosen to be larger than the feedback delay $R$, then the following discrete map can be constructed:

$$
\mathbf{z}(K)=\Phi \mathbf{z}(0)
$$

where $\mathbf{z}(j), j=0, K$ is defined as in (3), and the coefficient matrix reads

$$
\boldsymbol{\Phi}=\left(\begin{array}{ccccc}
\mathbf{M} & \mathbf{A}^{K-R} \mathbf{B} & \mathbf{A}^{K-R+1} \mathbf{B} & \ldots & \mathbf{A}^{R-1} \mathbf{B} \\
\mathbf{0} & \mathbf{0} & \mathbf{0} & \ldots & \mathbf{0} \\
\vdots & \vdots & \vdots & & \vdots \\
\mathbf{0} & \mathbf{0} & \mathbf{0} & \ldots & \mathbf{0}
\end{array}\right)
$$

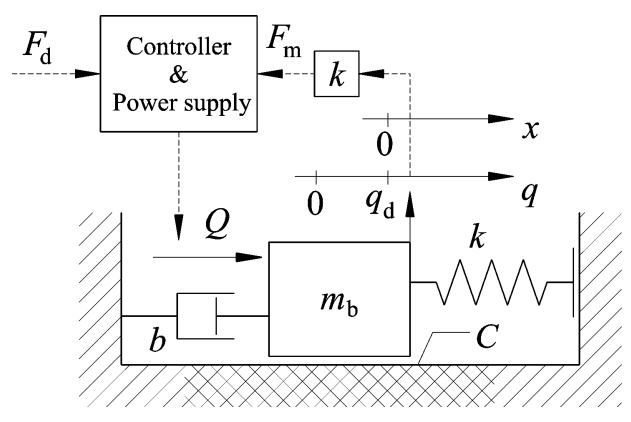

Fig. 1. Mechanical model of the force control process.

with

$$
\mathbf{M}=\mathbf{A}^{K}+\mathbf{A}^{K-R-1} \mathbf{B D} .
$$

Clearly, $m R$ eigenvalues of $\boldsymbol{\Phi}$ are zero, while its nonzero eigenvalues are just equal to the eigenvalues of the $n \times n$ matrix M. Consequently, stability properties are determined only by $n$ poles instead of $(n+m R)$ ones. Moreover, if the pair $\left(\mathbf{A}^{K}, \mathbf{A}^{K-R-1} \mathbf{B}\right)$ is controllable, then the poles of the system can arbitrarily be placed.

From now on, controller (5) will be called the act-and-wait controller, while controller (2), where the control is continuously active, is called the continuous controller.

\section{Force CONTROL MODEL}

The mechanical model of a single DOF force control process is shown in Fig. 1. Here, the modal mass $m_{\mathrm{b}}$ and the equivalent stiffness $k$ represent the inertia and the stiffness of the robot and the environment, while equivalent damping $b$ models the viscous damping originated from the servo motor characteristics and the environment. Variable $q$ denotes the position of the robot, while $x$ is a small perturbation around the desired position $q_{\mathrm{d}}=F_{\mathrm{d}} / k$ with $F_{\mathrm{d}}$ denoting the desired contact force. Force $Q$ represents the controller's action and $C$ is the magnitude of the effective Coulomb friction.

Considering a proportional-differential force controller, the control force can be given as

$$
Q(t)=F_{\mathrm{d}}-P\left(F_{\mathrm{m}}(t)-F_{\mathrm{d}}\right)-D\left(\dot{F}_{\mathrm{m}}(t)-\dot{F}_{\mathrm{d}}\right)
$$

where $P$ is the proportional gain, $D$ is the differential gain, $F_{\mathrm{d}}$ is the desired force, and $F_{\mathrm{m}}$ is the measured force. This type of control force computation was also considered in [4]. The equation of motion reads

$$
\begin{aligned}
m_{\mathrm{b}} \ddot{q}(t)+b \dot{q}(t)+k q(t) & =F_{\mathrm{d}}-P\left(F_{\mathrm{m}}(t)-F_{\mathrm{d}}\right) \\
& -D\left(\dot{F}_{\mathrm{m}}(t)-\dot{F}_{\mathrm{d}}\right)-C \operatorname{sgn} \dot{q}(t) .
\end{aligned}
$$

Assuming steady-state condition by setting all the time derivatives to zero, considering a constant Coulomb friction force, and using $F_{\mathrm{m}}=k q(t)$, the maximum force error can be given as

$$
F_{\mathrm{e}}^{\max }=\frac{C}{1+P}
$$

(see, e.g., [4], and [5]). Thus, the higher the proportional gain $P$, the less the force error, while the differential gain $D$ has no 
effect on the accuracy of the force control process. Integral control can also be used to compensate steady-state force error in constant velocity applications. However, if the system trajectory encounters velocity reversal, then simple integral control rather increases than reduces frictional disturbance. Since the steadystate force error is always within the deadband $\left[-F_{\mathrm{e}}^{\max }, F_{\mathrm{e}}^{\max }\right]$, it can essentially be reduced by reducing $F_{\mathrm{e}}^{\max }$. This can be achieved by increasing the stiffness or by increasing the proportional gain $P$, which is a kind of artificial stiffness in the system. Theoretically, there is no upper limit for the proportional gain $P$, since the constant solution $q(t) \equiv q_{\mathrm{d}}$ of (11) is always asymptotically stable when $C=0$. Experiments show, however, that the real system is not stable for large proportional gains [16]. This instability is caused by the digital effects and the corresponding delays in the feedback loop.

In the next sections, mathematical models for the continuous and for the act-and-wait controller are presented in case of digital control, and the corresponding stability properties are compared using stability charts. Since the force error does not depend on the differential gain $D$, a pure proportional controller is investigated. Thus, the only control parameter is $P$, while $D=0$.

\section{A. Digital Control With Continuous Controller}

In digital control, the control force is updated in discrete instants such that

$$
\begin{aligned}
Q(t) & =F_{\mathrm{d}}-P\left(F_{\mathrm{m}}\left(t_{j-1}\right)-F_{\mathrm{d}}\right) \\
& =k q_{\mathrm{d}}-P\left(k q\left(t_{j-1}\right)-k q_{\mathrm{d}}\right), \quad t \in\left[t_{j}, t_{j+1}\right)
\end{aligned}
$$

where $t_{j}=j \Delta t, j=0,1,2, \ldots$, and $\Delta t$ is the sampling time. Here, we assume that the sampling frequency of the force sensor and the frequency of the digital control are both $f_{\mathrm{s}}=1 / \Delta t$, and the data processing and the control computation are executed within a single sampling period. Thus, the control force $Q$ commanded over the sampling period $\left[t_{j}, t_{j+1}\right)$ is computed using the contact force measured at instant $t_{j-1}$. This presents a short delay $\tau=\Delta t$ in the feedback loop, in addition to the zero-order hold. The corresponding equation of motion reads

$$
\begin{aligned}
m_{\mathrm{b}} \ddot{q}(t)+b \dot{q}(t)+k q(t)= & k q_{\mathrm{d}}-P\left(k q\left(t_{j-1}\right)-k q_{\mathrm{d}}\right) \\
& -C \operatorname{sgn} \dot{q}(t), \quad t \in\left[t_{j}, t_{j+1}\right) .
\end{aligned}
$$

Stability properties of the system can be given by analyzing the variational system around the desired motion $q_{\mathrm{d}}$. For this computation, we neglect the dry friction from the model. Considering that $q(t)=q_{\mathrm{d}}+x(t)$, the variational system reads

$$
\ddot{x}(t)+2 \zeta \omega_{\mathrm{n}} \dot{x}(t)+\omega_{\mathrm{n}}^{2} x(t)=-\omega_{\mathrm{n}}^{2} P x\left(t_{j-1}\right), \quad t \in\left[t_{j}, t_{j+1}\right)
$$

where $\omega_{\mathrm{n}}=\sqrt{k / m_{\mathrm{b}}}$ is the natural angular frequency of the uncontrolled undamped system, and $\zeta=b /\left(2 m_{\mathrm{b}} \omega_{\mathrm{n}}\right)$ is the damping ratio. We introduce the frequency ratio

$$
\alpha=f_{\mathrm{n}} / f_{\mathrm{s}}
$$

where $f_{\mathrm{n}}=\omega_{\mathrm{n}} /(2 \pi)$ is the natural frequency of the uncontrolled undamped system and $f_{\mathrm{s}}=1 / \Delta t$ is the sampling fre- quency. Rescaling the time such that $\tilde{t}=t / \Delta t$ yields

$$
\begin{aligned}
\ddot{x}(\tilde{t})+4 \pi \zeta \alpha \dot{x}(\tilde{t}) & +4 \pi^{2} \alpha^{2} x(\tilde{t}) \\
= & -4 \pi^{2} \alpha^{2} P x(j-1), \quad \tilde{t} \in[j, j+1) .
\end{aligned}
$$

Thus, the system is characterized by three dimensionless parameters, the relative damping $\zeta$, the proportional gain $P$, and the frequency ratio $\alpha$. This way, a wide range of system/environment combinations can be described by analyzing different values of $\zeta, P$, and $\alpha$.

Equation (17) can be transformed into the state-space form

$$
\dot{\mathbf{x}}(\tilde{t})=\widehat{\mathbf{A}} \mathbf{x}(\tilde{t})+\widehat{\mathbf{B}} \widehat{\mathbf{D}} \mathbf{x}(j-1), \quad \tilde{t} \in[j, j+1)
$$

with

$$
\begin{aligned}
\mathbf{x}(\tilde{t}) & =\left(\begin{array}{c}
x(\tilde{t}) \\
\dot{x}(\tilde{t})
\end{array}\right), \quad \widehat{\mathbf{A}}=\left(\begin{array}{cc}
0 & 1 \\
-4 \pi^{2} \alpha^{2} & -4 \pi \zeta \alpha
\end{array}\right) \\
\widehat{\mathbf{B}} & =\left(\begin{array}{c}
0 \\
-4 \pi^{2} \alpha^{2}
\end{array}\right), \quad \widehat{\mathbf{D}}=\left(\begin{array}{ll}
P & 0
\end{array}\right) .
\end{aligned}
$$

Solving (18) over a unit sampling period $(\Delta \tilde{t}=1$ on the rescaled time domain) results in a discrete system of form (1) and (2) with matrices

$$
\mathbf{A}=\mathrm{e}^{\hat{\mathbf{A}}}, \quad \mathbf{B}=\left(\mathrm{e}^{\hat{\mathbf{A}}}-\mathbf{I}\right) \widehat{\mathbf{A}}^{-1} \widehat{\mathbf{B}}, \quad \mathbf{D}=\widehat{\mathbf{D}}
$$

and with feedback delay $R=1$. In this case, $n=2$ and $m=1$; thus, the stability of the system can be assessed by checking all the $(n+m R)=3$ eigenvalues of the monodromy matrix $\Psi$ given in the form (4).

\section{B. Digital Control With Act-and-Wait Controller}

The control force associated with the act-and-wait control concept can be given as

$$
Q_{\mathrm{a} \& \mathrm{w}}(t)=F_{\mathrm{d}}-g(j) P\left(F_{\mathrm{m}}\left(t_{j-1}\right)-F_{\mathrm{d}}\right), \quad t \in\left[t_{j}, t_{j+1}\right)
$$

where $g(j)$ is the $K$-periodic act-and-wait switching function defined in (6), i.e.

$$
Q_{\mathrm{a} \& \mathrm{w}}(t)= \begin{cases}F_{\mathrm{d}}-P\left(F_{\mathrm{m}}\left(t_{j-1}\right)-F_{\mathrm{d}}\right), & \text { if } j=h K, h \in \mathbb{Z} \\ F_{d}, & \text { otherwise. }\end{cases}
$$

This means that for $(K-1)$ number of steps, the control force is just equal to the desired force $F_{\mathrm{d}}$, and the feedback is switched on only in each $K$ th step. The corresponding variational system on the rescaled time domain reads

$$
\begin{aligned}
& \ddot{x}(\tilde{t})+4 \pi \zeta \alpha \dot{x}(\tilde{t})+4 \pi^{2} \alpha^{2} x(\tilde{t}) \\
& =-g(j) 4 \pi^{2} \alpha^{2} P x(j-1), \quad \tilde{t} \in[j, j+1) .
\end{aligned}
$$

This equation can be transformed into the state-space form

$$
\dot{\mathbf{x}}(\tilde{t})=\widehat{\mathbf{A}} \mathbf{x}(\tilde{t})+g(j) \widehat{\mathbf{B}} \widehat{\mathbf{D}} \mathbf{x}(j-1), \quad \tilde{t} \in[j, j+1)
$$

with $\mathbf{x}(\tilde{t}), \widehat{\mathbf{A}}, \widehat{\mathbf{B}}, \widehat{\mathbf{D}}$ given as in (18). Solution of (23) over a sampling period gives a discrete system that is equivalent to (1) and (5) with matrices $\mathbf{A}, \mathbf{B}$, and $\mathbf{D}$ defined as in (19), and with feedback delay $R=1$. 
The results for system (1) with the act-and-wait controller (5) can now be applied to the presented force control problem: if the period parameter $K$ is larger than the feedback delay $R=1$, then the system has only $n=2$ poles that are the eigenvalues of matrix $\mathbf{M}$ defined in (9). The obvious choice for the period parameter is $K=2$. Note that complete pole placement is still not possible in this case, since the number of poles to be controlled is 2 , and the only control parameter is the proportional gain $P$; still, the stability properties of the system improve significantly, as shown in the next section.

\section{Comparison of Continuous and Act-and-Wait Controller}

Stability of the control processes can be determined by the analysis of the characteristic multipliers that are the eigenvalues of matrix $\Psi$ in (4) for the continuous controller, and the eigenvalues of matrix $\mathbf{M}$ in (9) for the act-and-wait controller. The stability properties can be represented by stability charts in the plane of the frequency ratio $\alpha=f_{\mathrm{n}} / f_{\mathrm{s}}$ and the proportional gain $P$. In addition to stability charts, the frequencies of the vibration that arise at the stability boundaries can also be determined using the phase angle of the critical (largest in modulus) eigenvalues of $\Psi$ and $M$, respectively. Since vibration frequencies can easily be determined experimentally, they can also be used to verify the theoretical predictions [16].

The vibration frequencies can be obtained by the analysis of (17) and (22). Due to the sampling, (17) is periodic at the sampling period, $\widetilde{T}=1$, while $(22)$ is periodic at the act-andwait period, $\widetilde{T}=K$. Here, $\widetilde{T}$ denotes the principal period of the system on the rescaled time domain, i.e., $\widetilde{T}=T / \Delta t$. Vibrations arise when the system loses stability; therefore, we assume that the critical characteristic multiplier satisfies $\left|\mu_{1}\right|=1$. Note that $\mu_{1}$ may be either real \pm 1 or complex $\mathrm{e}^{ \pm \mathrm{i} \omega_{1}}$. According to the Floquet theory, the solution corresponding to the characteristic multiplier $\mu_{1}$ reads

$$
x(\tilde{t})=p(\tilde{t}) e^{\lambda_{1} \tilde{t}}+\bar{p}(\tilde{t}) e^{\bar{\lambda}_{1} \tilde{t}}
$$

where $p(\tilde{t})$ is a $\widetilde{T}$-periodic function, the bar denotes complex conjugate, and $\lambda_{1}$ is the characteristic exponent, i.e., $\mu_{1}=e^{\lambda_{1} \widetilde{T}}$. Fourier expansion of $p(\tilde{t})$ and substitution of $\lambda_{1}=\mathrm{i} \omega_{1}$ result in

$$
x(\tilde{t})=\sum_{h=-\infty}^{\infty}\left(C_{h} \mathrm{e}^{\mathrm{i}\left(\omega_{1}+h 2 \pi / \widetilde{T}\right) \tilde{t}}+\bar{C}_{h} \mathrm{e}^{-\mathrm{i}\left(\omega_{1}+h 2 \pi / \widetilde{T}\right) \tilde{t}}\right)
$$

where $C_{h}$ and $\bar{C}_{h}$ are complex coefficients. Note that $\omega_{1}$ equals the phase angle describing the direction of $\mu_{1}$ on the complex plane so that $-\pi<\omega_{1}<\pi$. The exponents in (25) give the angular frequency content of the motion. The corresponding frequencies on the rescaled time domain are

$$
\tilde{f}_{\mathrm{vib}}=f_{\mathrm{vib}} / f_{\mathrm{s}}= \pm \frac{\omega_{1}}{2 \pi}+\frac{h}{\widetilde{T}}, \quad h=0,1,2, \ldots
$$

Here, $\tilde{f}_{\text {vib }}$ is the vibration frequency on the rescaled time domain, $f_{\mathrm{vib}}$ is the vibration frequency on the regular time domain in hertz, and $f_{\mathrm{s}}=1 / \Delta t$ is the sampling frequency. In further analyses, the ratio $f_{\mathrm{vib}} / f_{\mathrm{s}}$ will be used; thus, control processes with different sampling rates can be compared. Of course, only the positive frequencies have physical meaning.
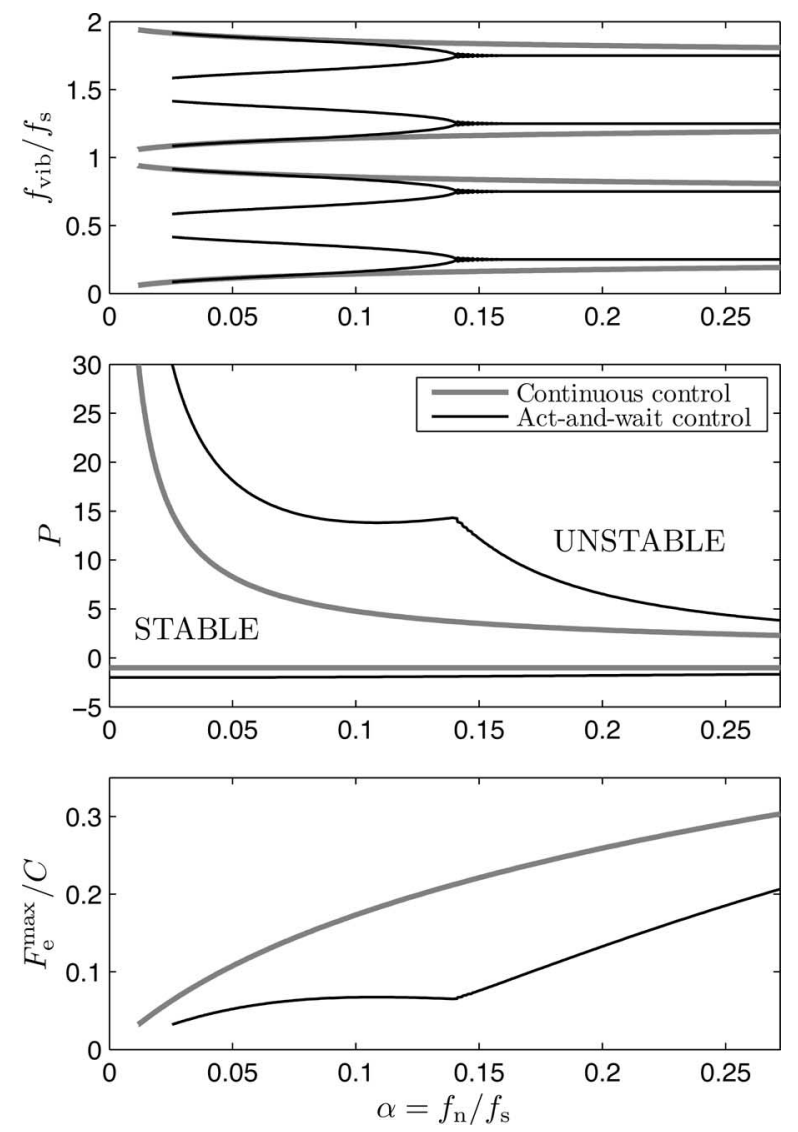

Fig. 2. Vibration frequencies (top), stability boundaries (middle), and the maximum force errors (bottom) for the continuous system (17) and for the actand-wait system (22) with period parameter $\widetilde{T}=K=2$. The relative damping is $\zeta=1.57$.

Fig. 2 presents the stability boundaries (middle panel), the associated vibration frequencies (top panel), the ratio of the maximum force error $F_{\mathrm{e}}$, and the Coulomb force $C$ (bottom panel) for the continuous control concept (thick gray) and for the actand-wait control concept (thin black). The stability charts were determined via point-by-point numerical evaluation of the critical eigenvalues of $\boldsymbol{\Psi}$ (for the continuous controller) and $\mathbf{M}$ (for the act-and-wait controller) over a $(200 \times 200)$-sized grid of parameters $\alpha$ and $P$. The period parameter for the act-and-wait controller was $\widetilde{T}=K=2$. It can be seen that there is an upper limit for the control gain $P$ for both cases. Above this stability boundary, the control process is unstable. The lower stability limits correspond to the negative proportional gain $P$ and have no practical relevance. The vibration frequencies in the top panel were determined according to (26) using the eigenvalues corresponding to the upper stability boundary. The ratio of the maximum force error $F_{\mathrm{e}}^{\max }$ and the Coulomb force $C$ is presented in the bottom panel in Fig. 2. According to (12), this ratio comes from the simple computation $F_{\mathrm{e}}^{\max } / C=1 /(1+P)$, where $P$ is the critical (maximum stable) proportional gain from the middle panel. Fig. 2 shows that the critical proportional gains are essentially larger for the act-and-wait controller than for the continuous controller, and the corresponding force error is smaller by a factor of 2-3.

Analysis of the eigenvalues shows that the continuous control concept loses stability with a complex pair of characteristic 
TABLE I

SAMPLING FREQUENCIES DURING THE TESTS

\begin{tabular}{|c|c|c|c|c|c|c|c|c|c|c|}
\hline test & a & b & c & d & e & f & g & h & i & j \\
\hline$\Delta t[\mathrm{~ms}]$ & 10 & 20 & 30 & 40 & 50 & 60 & 70 & 80 & 90 & 100 \\
\hline$f_{\mathrm{s}}[\mathrm{Hz}]$ & 100 & 50 & 33.33 & 25 & 20 & 16.67 & 14.29 & 12.5 & 11.11 & 10 \\
\hline$\alpha$ & 0.02474 & 0.04948 & 0.07422 & 0.09896 & 0.1237 & 0.1484 & 0.1732 & 0.1979 & 0.2227 & 0.2474 \\
\hline
\end{tabular}

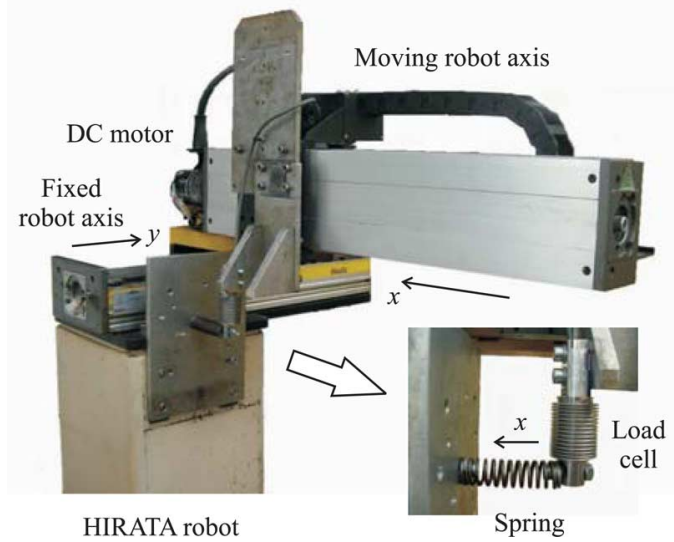

Fig. 3. Experimental setup.

multipliers that corresponds to a secondary Hopf bifurcation of the underlying nonlinear system. In this case, quasi-periodic vibrations arise.

For the act-and-wait control case, if $f_{\mathrm{n}} / f_{\mathrm{s}}>0.14$, then the critical eigenvalue is real, and it crosses the unit circle at -1 that corresponds to flip (or period doubling) bifurcation of the underlying nonlinear system. In this case, the period of the arising vibrations is just double of the act-and-wait period. If $f_{\mathrm{n}} / f_{\mathrm{s}}<0.14$, then the secondary Hopf bifurcation occurs similarly to the continuous control case. The differences between the secondary Hopf and flip cases can clearly be seen in the frequency diagram as well.

\section{EXPERIMENTAL VALIDATION}

For the experimental validation of the theoretical results, force control process was implemented using a HIRATA (MB-H180500) dc drive robot shown in Fig. 3. This robot has two linear axes. The first axis (the $y$-direction) was fixed during the experiments, while the second axis (the $x$-direction) was connected to the base of the robot (environment) by a helical spring of stiffness $k=7144 \mathrm{~N} / \mathrm{m}$. The contact force between the environment and the spring was induced by the displacement of the moving robot axis in the $x$-direction. The force was measured by a Tedea-Huntleight Model 355 load cell mounted between the spring and the robot's flange. The driving system of the moving axis consisted of a HIRATA HRM-020-100-A de servo motor connected directly to a ballscrew with a $20-\mathrm{mm}$ pitch thread. The robot was controlled by a microcontroller-based control unit providing the maximum sampling frequency of $1 \mathrm{kHz}$ for the overall force control loop. The control force was set proportionally to the measured force error by the pulse with modulation of the supply voltage of the dc motor. The controller made it possible to vary the sampling time and the time delay as integer multiples of $1 \mathrm{~ms}$, and to set the proportional gain and the desired contact force arbitrarily. The modal mass and the damping ratio of the system were experimentally determined: $m_{\mathrm{b}}=29.57 \mathrm{~kg}$ and $b=1447 \mathrm{Ns} / \mathrm{m}$. The natural angular frequency of the uncontrolled undamped system was $\omega_{\mathrm{n}}=\sqrt{k / m_{\mathrm{b}}}=15.54 \mathrm{rad} / \mathrm{s}$, the damping ratio was $\zeta=b /\left(2 m_{\mathrm{b}} \omega_{\mathrm{n}}\right)=1.57$. The Coulomb friction originated mostly from the dc drive system and was measured to be $C=16.5 \mathrm{~N}$.

During the tests, the desired contact force was set to $F_{\mathrm{d}}=$ $50 \mathrm{~N}$. Due to the relatively small stiffness, the system is characterized by a well-defined single natural frequency: $f_{\mathrm{n}}=$ $\omega_{\mathrm{n}} /(2 \pi)=2.474 \mathrm{~Hz}$. The disturbing effect of higher order modes can, therefore, be neglected, and a single DOF model can be used. Since the natural frequency of the system was constant during the tests, different frequency ratios $\alpha=f_{\mathrm{n}} / f_{\mathrm{s}}=f_{\mathrm{n}} \Delta t$ were attained by changing the sampling time $\Delta t$ between 10 and $100 \mathrm{~ms}$ according to Table I. For each sampling period, the proportional gain $P$ was increased step by step. The control process was declared unstable if the robot started oscillations for perturbations larger than $50 \mathrm{~N}$. In most cases, the experimental stability boundaries were clearly identified.

Fig. 4 presents the comparison of the theoretical and the experimental results for the continuous control case. Left middle panel shows the theoretically predicted stability boundaries (lines) and the experimentally determined ones (crosses). In order to verify unstable behavior, the frequency spectra of the arising vibrations were compared to the theoretical predictions. Left top panel presents the theoretically predicted vibration frequencies according to (26). Here, the dots denote the theoretical frequencies for the test points a, b, c, etc. Right panels show the corresponding power spectra density (PSD) diagrams that were determined using the recorded time history of the measured contact force. Here, the frequencies that were predicted theoretically are denoted by the black dots for reference. In the PSD diagrams, logarithmic scale is used for the vertical axes in order to show the higher frequencies clearly. Thus, the structure of the frequency diagrams can be compared to the theoretical predictions. Left bottom panels show the ratio of the maximum force error $F_{\mathrm{e}}^{\max }$ and the Coulomb friction force $C$. The theoretically predicted envelope curve for this ratio is denoted by the line, while the experimentally measured values are denoted by the crosses. It can be seen that the measured force errors are smaller than the predicted maximum. Fig. 4 shows that the theoretical predictions for the continuous control case are verified by the experiments regarding all the stability boundaries, the structure of the vibration frequencies, and the force error.

Fig. 5 presents the same comparison of the theoretical and the experimental results for the act-and-wait control case. Left middle panel shows the stability boundaries, left top panel shows the theoretically predicted vibration frequencies, and right 

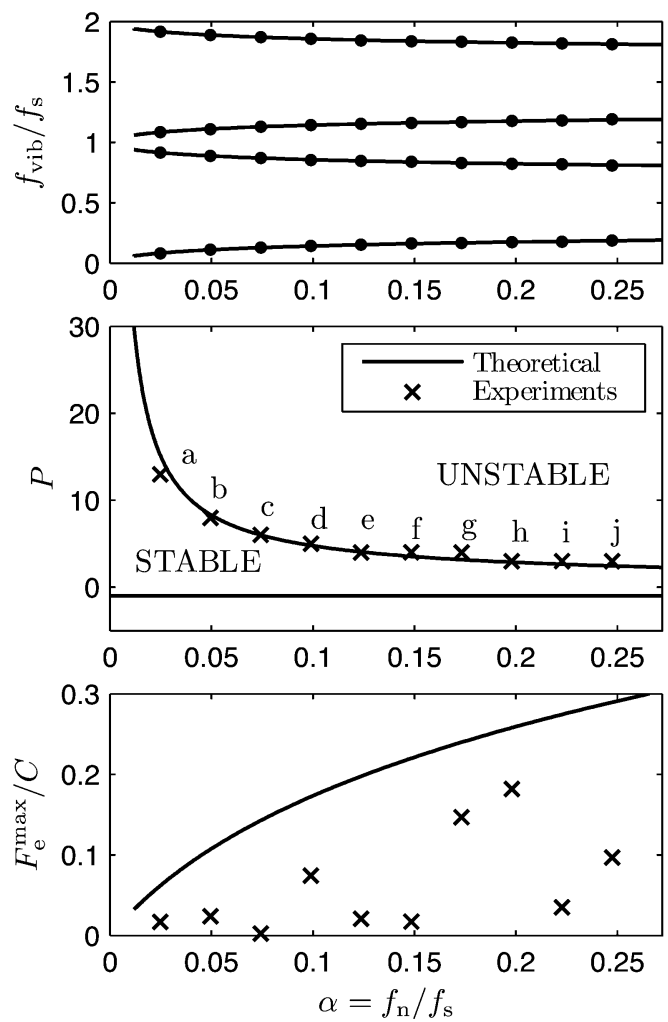
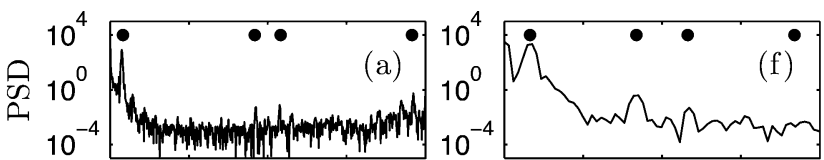

$\begin{array}{llllllllll}0 & 0.5 & 1 & 1.5 & 2 & 0 & 0.5 & 1 & 1.5 & 2\end{array}$

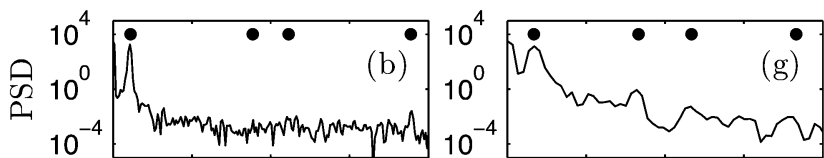

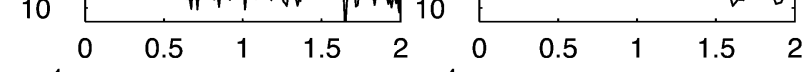

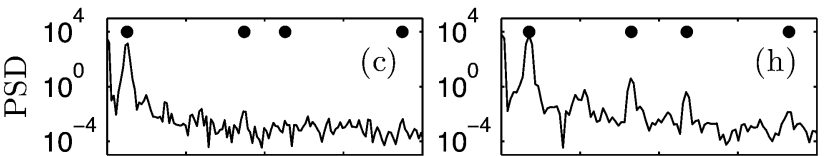

$\begin{array}{llllllllll}0 & 0.5 & 1 & 1.5 & 2 & 0 & 0.5 & 1 & 1.5 & 2\end{array}$
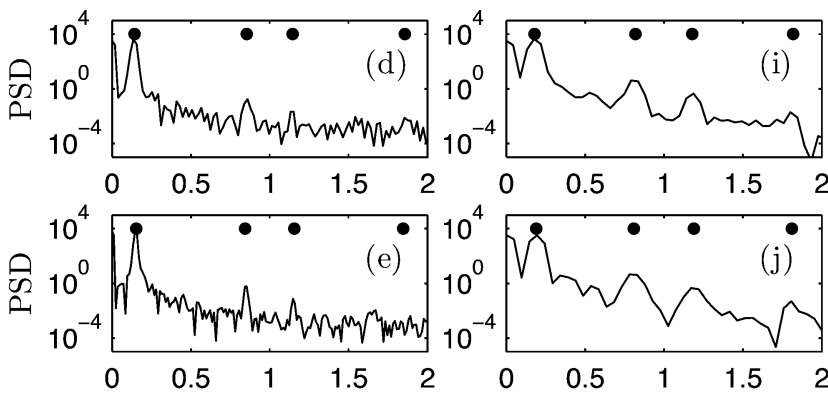

$f_{\mathrm{vib}} / f_{\mathrm{s}}$ (i)

Fig. 4. Experimental and theoretical stability charts (left middle), theoretical vibration frequencies (left top), force errors (left bottom), and experimental PSD diagrams (right) for the continuous control concept.
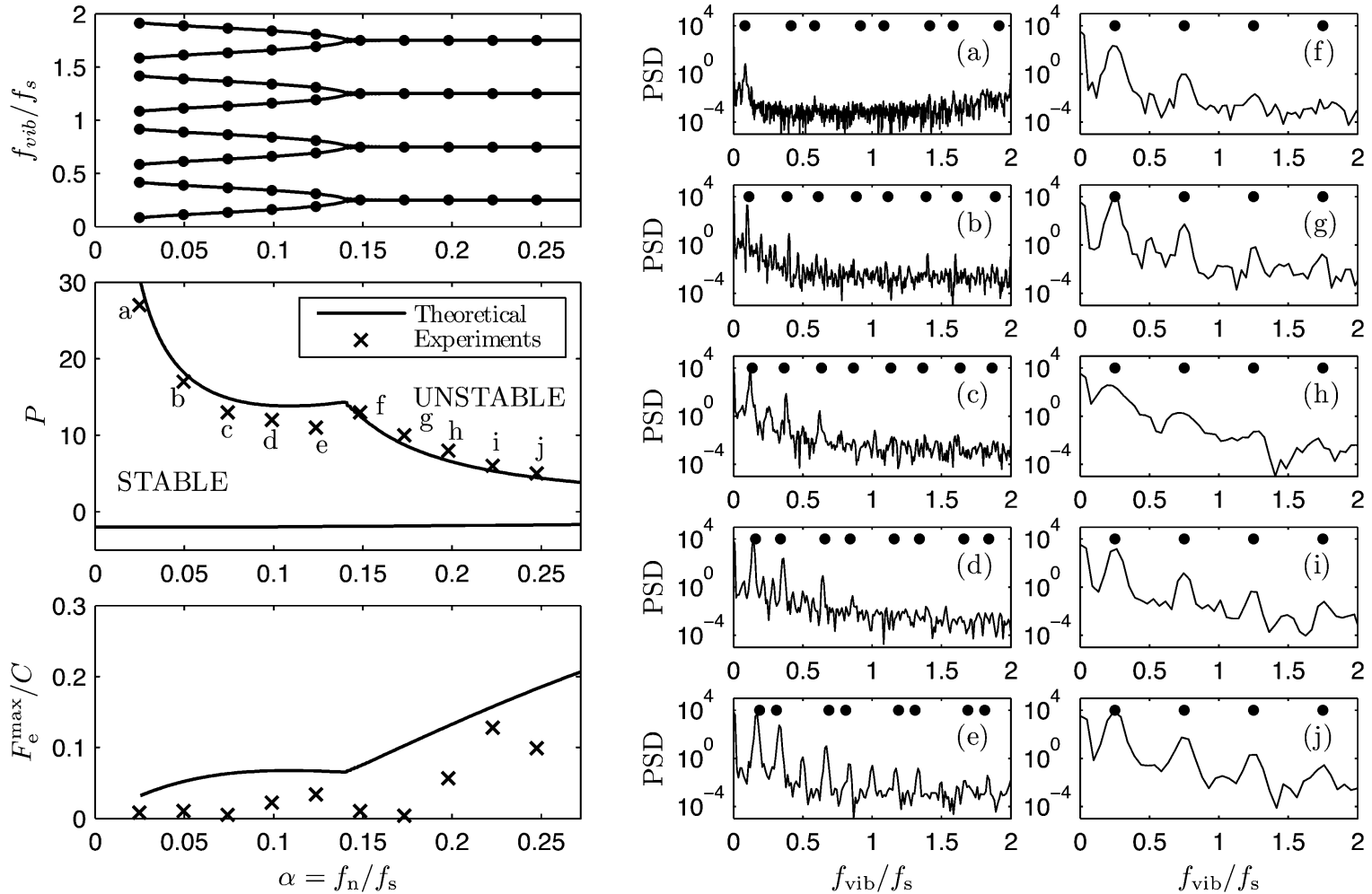

Fig. 5. Experimental and theoretical stability charts (left middle), theoretical vibration frequencies (left top), force errors (left bottom), and experimental PSD diagrams (right) for the act-and-wait control concept. 

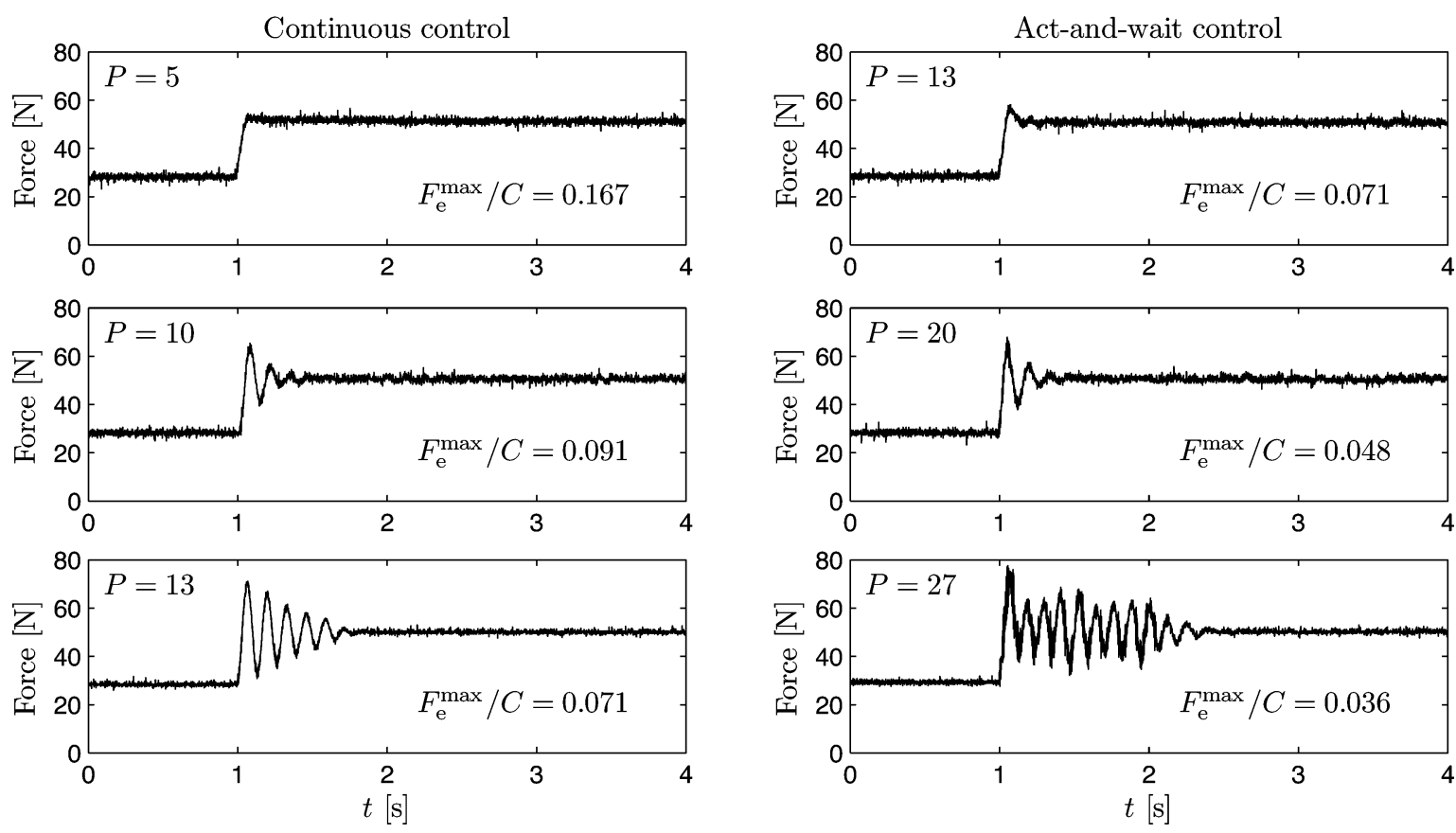

Fig. 6. Time histories of the measured contact force for $\Delta t=10 \mathrm{~ms}$.

panels present the measured PSD diagrams. The predicted and the measured force errors are presented in the left bottom panel. It can be seen that the experimental results agree well with the theoretical predictions for the act-and-wait controller as well.

The results shown in Figs. 4 and 5 confirm that larger proportional gains can be used with stable control process if the actand-wait concept is used instead of the continuous controller. Consequently, the force error can be reduced by a factor of 2-3.

The gain of the act-and-wait method is demonstrated in Fig. 6, where time histories of the measured contact force are presented for different control concepts with the same sampling period $\Delta t=10 \mathrm{~ms}$. The controller is switched on at $t=1 \mathrm{~s}$ for all the six cases. Left panels present the continuous control case for proportional gains $P=5,10$, and 13 . Case $P=13$ corresponds to point "a" in the stability chart of Fig. 4. For $P=13.5$, the control process was found to be unstable. The ratio $F_{\mathrm{e}}^{\max } / C=$ $1 /(1+P)$ is also presented in each panel in order to show the tendency of the force error for increasing $P$. It can be seen that the overshoot increases with the proportional gain, while the force error decreases.

For the act-and-wait controller, the proportional gain can be increased up to $P=27$ without losing stability. Right panels in Fig. 6 present the act-and-wait control case for proportional gains $P=13,20$, and 27 . The case $P=27$ corresponds to point "a" in the stability chart of Fig. 5. For $P=27.5$, the control process was found to be unstable. The tendency of the overshoot and the maximum force error for increasing proportional gains are similar to those of the continuous controller: The overshoot increases, while the force error decreases for increasing $P$. However, for the same proportional gain, the overshoot is significantly smaller for the act-and-wait controller than it is for the continuous controller. This can clearly be seen in Fig. 6 for the proportional gain $P=13$ (left bottom and right top panels). This is due to the fact that the continuous control system with $P=13$ is close to the stability boundary (for $P=13.5$, the system is already unstable), while the act-and-wait control system is stable up to $P=27$. For the act-and-wait controller, the force error can be decreased further by increasing the proportional gain, but in this case, the overshoot increases. Still, in some applications, it might be acceptable to have a strong transient with a relatively large overshoot in order to provide a minimal force error during the steady state of the system.

In Fig. 6, the transient behavior of the system can also be seen. For the continuous controller with $P=13$, the transient vibrations decay almost linearly in time, which refers to the presence of Coulomb friction. The frequencies appearing in the transient can be identified using panel (a) in Fig. 4. These frequencies are 8.4, 91.6, 108.4, and $191.6 \mathrm{~Hz}$. Here, the lowest frequency, $8.4 \mathrm{~Hz}$, is the dominant frequency that clearly appears in the force signal in Fig. 6 as well. For the act-and-wait controller with $P=27$, the transient vibrations decay in a quasi-periodic way due to the periodic switching of the controller. The corresponding frequencies can be read from panel (a) in Fig. 5 as 8.5, $41.5,58.5,91.5,108.5$, and $141.5 \mathrm{~Hz}$.

\section{CONCLUSION}

The act-and-wait control concept was applied to an experimental digital force control process with a short (one sample unit) feedback delay. The crux of the concept is that the feedback loop is switched off and on periodically during the control process so that the duration of the switch-off period is larger than the feedback delay. The experimental setup was designed such that the control gain can be varied in each sampling; thus, the act-and-wait concept can be implemented in the system. The technique was compared to the traditional, continuous control case, when the feedback loop is continuously active.

Stability charts were constructed that plot the critical proportional gains, where the process loses stability, as a function of the frequency ratio $\alpha=f_{\mathrm{n}} / f_{\mathrm{s}}$ with $f_{\mathrm{n}}$ being the natural 
frequency of the uncontrolled undamped system and $f_{\mathrm{s}}$ being the sampling frequency. It was shown that the application of the act-and-wait concept allows the use of larger proportional gains without losing stability: the critical gains for the act-and-wait controller are about double/triple of the critical gains associated with the continuous controller. Consequently, the force error can significantly be decreased by the application of the act-and-wait control concept. The theoretical results were confirmed by experiments for a range of frequency ratios. Vibration frequencies at the stability boundaries were used to verify the model. The theoretically predicted frequencies agreed well with the experimentally determined PSD diagrams.

\section{REFERENCES}

[1] D. E. Whitney, "Force feedback control of manipulator fine motion," Trans. ASME, J. Dyn. Syst., Meas. Control, vol. 98, pp. 91-97, 1977.

[2] M. T. Mason, "Compliance and force control for computer controlled manipulators," IEEE Trans. Syst., Man, Cybern., vol. SMC-11, no. 6, pp. 418-432, Jun. 1981.

[3] M. H. Raibert and J. J. Craig, "Hybrid position/force control of manipulators," Trans. ASME, J. Dyn. Syst. Meas. Control, vol. 102, pp. 126-133, Jun. 1981.

[4] J. J. Craig, Introduction to Robotics Mechanics and Control. Reading, MA: Addison-Wesley, 1986.

[5] H. Asada and J.-J. E. Slotine, Robot Analysis and Control. New York: Wiley, 1986

[6] C. Canudas, B. Siciliano, and G. Bastin, Theory of Robot Control. New York: Springer-Verlag, 1996.

[7] D. M. Gorinevsky, A. M. Formalsky, and A. Y. Schneider, Force Control of Robotics Systems. Boca Raton, FL: CRC Press, 1997.

[8] G. Stépán, "Vibrations of machines subjected to digital force control," Int. J. Solids Struct., vol. 38, no. 10-13, pp. 2149-2159, Mar. 2001.

[9] S. Munir and W. J. Book, "Internet-based teleoperation using wave variables with prediction," IEEE/ASME Trans. Mechatronics, vol. 7, no. 2, pp. 124-133, Jun. 2002

[10] I. G. Polushin, P. X. Liu, and C.-H. Lung, "A force-reflection algorithm for improved transparency in bilateral teleoperation with communication delay,” IEEE/ASME Trans. Mechatronics, vol. 12, no. 3, pp. 361-374, Jun. 2007.

[11] C. Cho, J.-B. Song, and M. Kim, "Stable haptic display of slowly updated virtual environment with multirate wave transform," IEEE/ASME Trans. Mechatronics, vol. 13, no. 5, pp. 566-575, Oct. 2008

[12] T. Insperger and G. Stépán, "Act-and-wait control concept for discretetime systems with feedback delay," IET_Control Theory Appl., vol. 1, no. 3, pp. 553-557, May 2007.

[13] T. Insperger, "Act-and-wait concept for time-continuous control systems with feedback delay," IEEE Trans. Control Syst. Technol., vol. 14, no. 5, pp. 974-977, Sep. 2006.

[14] B. C. Kuo, Digital Control Systems. Champaign, IL: SRL, 1977.

[15] K. J. Aström and B. Wittenmark, Computer Controlled Systems: Theory and Design. Englewood Cliffs, NJ: Prentice-Hall, 1984.

[16] G. Stépán, A. Steven, and L. Maunder, "Design principles of digitally controlled robots," Mech. Mach. Theory, vol. 25, no. 5, pp. 515-527, 1990.

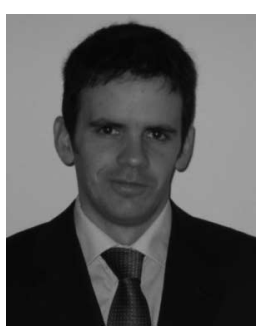

Tamás Insperger was born in Hódmezovásárhely, Hungary, in 1976. He received the M.Sc. and Ph.D degrees in mechanical engineering from the Budapest University of Technology and Economics (BME), Budapest, Hungary, in 1999 and 2002, respectively.

From 2003 to 2005, he held a Zoltán Magyary Postdoctoral Scholarship of the Foundation for Hungarian Higher Education and Research. He is currently an Associate Professor in the Department of Applied Mechanics, BME. His current research interests include dynamics and stability of time-delayed and periodic time-varying systems with applications to machine tool vibrations and feedback control systems.

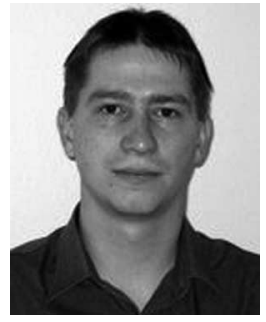

László L. Kovács was born in Budapest, Hungary, in 1975. He received the B.Sc. degree in mechanical engineering and the M.Sc. and Ph.D. degrees in applied mechanics from the Budapest University of Technology and Economics (BME), Budapest, in 1998, 2000, and 2007, respectively.

He has been a Chemical and Food Industry Engineer. Since 2004, he has been a Research Associate in the Department of Applied Mechanics, BME. He is a member of the Hungarian Academy Research Group on Dynamics of Machines and Vehicles. His current research interests include dynamics of machines subjected to digital force control, modeling and simulation of constrained dynamic systems, motion control of robotic devices, and service robotics.

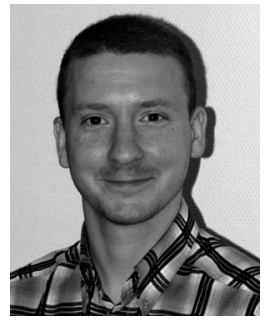

Péter Galambos was born in Györ, Hungary, in 1982 He received the M.Sc. degree in mechanical engineering in 2006 from the Budapest University of Technology and Economics (BME), Budapest, Hungary, where he is currently working toward the Ph.D. degree in the Department of Manufacturing Science and Technology.

He was a Research Intern at the Toshiba Corporate Research and Development Center during 20072008. His current research interests include telemanipulation, distributed robotic systems, middleware technologies, and cognitive informatics.

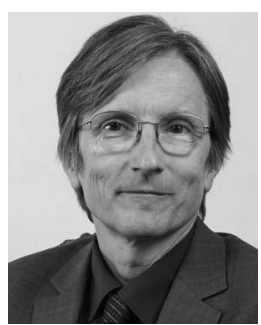

Gábor Stépán received the M.Sc. and Ph.D. degrees in mechanical engineering from the Technical University of Budapest, Budapest, Hungary, in 1978 and 1982, respectively, and the D.Sc. degree from the Hungarian Academy of Sciences, Budapest, Hungary, in 1994.

He was a Visiting Researcher in the Mechanical Engineering Department of the University of Newcastle upon Tyne, U.K., during 1988-1989, the Laboratory of Applied Mathematics and Physics of the Technical University of Denmark in 1991, and the Faculty of Mechanical Engineering of the Delft University of Technology during 1992-1993. He was a Fulbright Visiting Professor the Mechanical Engineering Department of the California Institute of Technology during 1994-1995, and a Visiting Professor the Department of Engineering Mathematics of Bristol University in 1996. He is currently a Professor of applied mechanics at the Budapest University of Technology and Economics, Budapest, where he is also the Dean of the Faculty of Mechancial Engineering. His current research interests include nonlinear vibrations in delayed dynamical systems, and applications in mechanical engineering and biomechanics such as wheel dynamics (rolling, braking, shimmy), robotic force control, machine tool vibrations, human balancing, and traffic dynamics.

Prof. Stépán has been a member of Euromech, the International Union of Theoretical and Applied Mechanics (IUTAM), and the Scientific Council of the International Center for Mechanical Sciences (CISM) since 2001. Between 1995 and 2003, he served as a member of the Executive Council of the International Federation for the Promotion of Mechanisms and Machines Sciences (IFToMM). He was elected as a member of the Hungarian Academy of Sciences in 2008. He is a member of the Editorial Boards of the Philosophical Transactions of the Royal Society A, the Journal of Nonlinear Science, the Journal of Vibration and Control, and Physica D. He is currently an Associate Editor of Mechanism and Machine Theory. 